\title{
The Shock of Aging: Molecular Chaperones and the Heat Shock Response in Longevity and Aging - A Mini-Review
}

\author{
Stuart K. Calderwood Ayesha Murshid Thomas Prince \\ Beth Israel Deaconess Medical Center, Harvard Medical School, Boston, Mass., USA
}

\begin{abstract}
Key Words
Heat shock protein - Aging - Aggregation - Molecular chaperone $\cdot$ CHIP $\cdot$ Ubiquitin $\cdot$ Proteasome $\cdot$ Heat shock factor 1
\end{abstract}

\begin{abstract}
Background: Aging can be thought of as the collision between destructive processes that act on cells and organs over the lifetime and the responses that promote homeostasis, vitality and longevity. However, the precise mechanisms that determine the rates of aging in organisms are not known. Objective: Macromolecules such as proteins are continuously exposed to potential damaging agents that can cause loss of molecular function and depletion of cell populations over the lifetime of essential organs. One of the key homeostatic responses involved in maintaining longevity is the induction of heat shock proteins (HSPs), a conserved reaction to damaged intracellular proteins. We aim to discuss how the interplay between protein damage and its repair or removal from the cell may influence longevity and aging. Methods: We have reviewed experiments carried out in mammalian and non-mammalian organisms on molecular chaperones and the transcription factor (heat shock factor 1, HSF1) responsible for their expression. We have discussed mechanisms through which these molecules are regulated in cells, respond to stimuli that enhance longevity and become impaired during aging. Results: The transcrip-
\end{abstract}

tion factor HSF1 initiates the prolific induction of HSP when cells are exposed to protein damage. HSPs are molecular chaperones that protect the proteome by folding denatured polypeptides and promoting the degradation of severely damaged proteins. Activation of HSF1 is coupled functionally to fundamental pathways of longevity and orchestrates the evasion of aging through HSP induction and antagonism of protein aggregation. In addition to mediating protein quality control, some HSPs such as Hsp27 and Hsp70 directly protect cells against damage-induced entry into death pathways. However, the heat shock response declines in potency over the lifetime, and enfeeblement of the response contributes to aging by permitting the emergence of protein aggregation diseases, reduction in cellular vigor and decreased longevity. Conclusions: Molecular chaperones play an important role in the deterrence of protein damage during aging and their expression is required for longevity. Chemical stimulation of HSP synthesis might therefore be a significant strategy in future design of antiaging pharmaceuticals.

Copyright $\odot 2009$ S. Karger AG, Basel

\section{Introduction: Molecular Mechanisms of Aging}

Aging is a complex process in which organisms undergo general or specific degeneration and death of cell populations within vital organs. It is not clear to what degree this decline is the result of inbuilt 'aging programs',

\section{KARGER}

() 2009 S. Karger AG, Basel

Fax +4161306 1234

E-Mail karger@karger.ch

www.karger.com
Accessible online at: www.karger.com/ger
Dr. Stuart K. Calderwood

BIDMC, 99 Brookline Avenue, Room 325E

Boston, MA 02215 (USA)

Tel. +1 617667 0970, Fax +1 6179755240

E-Mail scalderw@bidmc.harvard.edu 
to the wear and tear of day-to-day life or both processes. Genetic studies in model organisms show that two signaling pathways in particular significantly impact longevity. These include (1) activation of the Sir2 pathway and (2) a decline in signaling through the insulin/IGF$1 /$ mTOR pathway [1]. Sir2 is an NAD ${ }^{+}$-dependent histone deacetylase that catalyzes a key signaling pathway in cells involving the cleavage of acetyl groups from lysine residues in multiple substrates and its overexpression or induction in starvation leads to increased lifespan in the worm Caenorhabditis elegans and the fruit fly Drosophila melanogaster [1].

Aging and tissue degeneration involve the accumulation of damage to cellular macromolecules in non-dividing adult cells. Chemical damage due to oxidative stress, glycation and the addition of sugar residues has the capacity to modify both DNA and proteins, although the exact intracellular lesions that lead to loss of vigor and decreased lifespan are still undefined. It has been proposed recently that increased protein damage during aging may be exacerbated by a declining heat shock response, reduced levels of heat shock proteins (HSPs), and the resultant loss of protein quality control. These interactions will form the subject of this review.

\section{The Heat Shock Response}

Almost all prokaryotic and eukaryotic species exposed to elevated temperatures undergo production of a cohort of proteins in relatively massive amounts [2]. These proteins became known as HSPs and were shown to play a remarkably conserved role in homeostasis among all species [2]. The elevation in HSP levels during the heat shock response was shown to inhibit stress-mediated cell killing and recent experiments indicate a highly versatile role for these proteins as inhibitors of programmed cell death [3]. Intensive investigations then led to the elucidation of molecular functions of HSPs as molecular chaperones, proteins that cater to the tertiary structures of other proteins, known as their 'clients' [4].

\section{Protein Dysfunction and Aging}

Recent studies indicate that the heat shock response declines in aging cells and becomes enfeebled as organisms live beyond the mature adult stage. Age-dependent waning of the heat shock response is a general effect found in neuronal tissues [5-7], skeletal and cardiac mus- cle [8] and liver [9]. Cells lose the capacity to activate the transcriptional pathways leading to HSP synthesis. In neuronal tissues, decline in protein quality control was widely predicted, as the etiology of a number of diseases involve aggregation-prone proteins that form inclusion bodies whose occurrence is linked to pathology. These diseases include the most frequent neurodegenerative disorder, Alzheimer's disease, an ailment whose pathological symptoms are linked to accumulation of at least two types of inclusion formed from aggregation of the amyloid- $\beta$ peptide and the cytoskeletal protein tau [6]. In addition, Parkinson's disease is characterized by pathological inclusions containing another protein, $\alpha$-synuclein [6]. Further examples include the polyglutamine expansion diseases such as Huntington's disease and spinobulbar muscular atrophy in which proteins associated with disease pathology such as, respectively, huntingtin and androgen receptor contain areas of polyglutamine expansion. Polyglutamine repeat sequences promote a tendency to aggregation that correlates with repeat length. Pathology in each of these neuronal diseases involves loss of neurotransmission and cell death [7]. The striking characteristic in each of these disorders is that, although cells contain significant levels of aggregation-prone proteins, disease does not become established immediately but is instead observed as the mature individual ages [5]. This suggests the hypothesis that aging in the central nervous system and other tissues may involve time-dependent loss of protein quality control, a deficit that is permissive for aggregation and inclusion body formation. Such a decline would permit diseases linked to proteins such as amyloid- $\beta, \alpha$-synuclein and huntingtin, with little common physiological function except aggregation susceptibility, to induce neuronal cell killing by related mechanisms [6]. It is not clear why the central nervous system may be especially prone to protein aggregation/ inclusion body disorders, although the heat shock response seems to be particularly weakly expressed in these cells (discussed below).

Age-dependent decline in the heat shock response is also observed in muscle tissues. In these tissues, vigorous contraction leads to induction of HSP that is cytoprotective in nature [8]. These effects are severely blunted in the muscles of older animals and aging humans, suggesting that decline in muscle mass and force generation may be related to loss of HSP expression. Indeed chronic Hsp70 overexpression in transgenic mice partially reversed some of these effects [8]. The heat shock response is also known to protect cells against the toxic effects of alcohols, heavy metals, xenobiotics and oxidants [2]. This ho- 
Table 1. Mediators of protein quality control in the cytoplasm

\begin{tabular}{|c|c|c|c|}
\hline Protein & Function & Associated proteins & Ref. \\
\hline HSP27 & $\begin{array}{l}\text { Molecular } \\
\text { chaperone }\end{array}$ & None & $2,25,26$ \\
\hline HSP60 & Chaperonin & HSP10 & 2,4 \\
\hline HSP70 & $\begin{array}{l}\text { Molecular } \\
\text { chaperone }\end{array}$ & $\begin{array}{l}\text { HSP40, GrpE, BAG } \\
\text { HSPBP1, Hip, CHIP, Hop }\end{array}$ & $2,4,11$ \\
\hline HSC70 & $\begin{array}{l}\text { Molecular } \\
\text { chaperone }\end{array}$ & HSPBP1, Hip, Hop & $2,11,17$ \\
\hline HSP90 & $\begin{array}{l}\text { Molecular } \\
\text { chaperone }\end{array}$ & $\begin{array}{l}\text { p23, HOP } \\
\text { FKBP51, FKBP52 } \\
\text { Cyp40, Cdc37, p97 }\end{array}$ & $2,14,20$ \\
\hline HDAC6 & HDAC & (Hsp90, p97) & 19,20 \\
\hline SIRT1 & HDAC & (HSF1, tubulin) & 1,21 \\
\hline LAMP2A & $\begin{array}{l}\text { Membrane } \\
\text { receptor }\end{array}$ & $(\mathrm{Hsc} 70)$ & 17 \\
\hline HSF1 & $\begin{array}{l}\text { Transcription } \\
\text { factor }\end{array}$ & $\begin{array}{l}\text { hsp90, p23, FKBP } \\
\text { HDAC6, p97 }\end{array}$ & $30,33,34$ \\
\hline
\end{tabular}

Co-chaperones facilitate individual reactions between primary chaperones and unfolded clients. The co-chaperones HSP40, p23, FKBP51, 52, Cyp40 and Cdc37 facilitate interaction of Hsp90 with its clients. Other co-chaperones, including GrpE, BAG-1 and HSPBP1, function as nucleotide exchange factors that permit efficient ATP binding and hydrolysis by Hsp70. A third class of co-chaperones permits the molecular chaperones to interact with other proteins: Hop bridges the Hsp70 and Hsp90 systems and CHIP links Hsp70 to the ubiquitin-proteasome system. P97/VCP is an AAA ATPase discovered as an HDAC6 binding protein that is found associated with HSF1 and Hsp90. meostatic ability of HSP induction may be particularly significant in liver function. Indeed a decline in the heat shock response in aged livers has been a general finding and is associated with reduced abilities to respond to hepatic toxins such as carbon tetrachloride [9].

\section{Heat Shock Proteins and Protein Folding}

The HSP family includes the HSP10, HSP27, HSP40, HSP60, HSP70, HSP90, and HSP110 genes that encode molecular chaperones (table 1) [2, 10]. HSP10 and Hsp60 are classified as chaperonins, proteins that oligomerize to form large structures that can engulf their clients and function as folding chambers [10]. Molecular chaperones Hsp70 and Hsp90 bind to unfolded sequences in polypeptide substrates free in the cytoplasm, showing preference for hydrophobic regions [11]. These chaperones form large complexes containing accessory proteins (co-chaperones) that bind the primary chaperone in order to mediate client selection, ATP hydrolysis and cycles of association with and dissociation from the client [11]. Hsp70 and Hsp90 recognize and fold proteins and then are re- leased from such protein substrates accompanied by the hydrolysis of ATP catalyzed by the ATPase domains of the chaperones [11]. Folding of damaged proteins by Hsp70 is depicted in figure 1. Protein folding also involves a family of smaller HSPs, such as the chaperone Hsp27, which function by an alternate mechanism; highmolecular-weight complexes containing large numbers of such small HSPs mediate client holding and folding in an ATP-independent manner (fig. 1). Efficient folding of the proteome involves coordinated activity of each of these chaperone types.

Of key importance to the regulation of the Hsp70 and Hsp90 chaperone systems and protein quality control is the presence of binding sites at the extreme carboxy-terminus of each molecule for proteins that contain the tetratricopeptide repeat (TPR) domain $[11,12]$. TPR domain proteins that can bind Hsp70 and Hsp90 include scaffold protein Hop which possesses at least two TPR domains [12]. Hop can bind simultaneously both to Hsp70 and Hsp90 and, by stabilizing the interactions of these chaperones, permit coordinated activity in protein folding [11]. 
Fig. 1. Pathways of protein folding after exposure to protein damage during aging. Unfolded proteins accumulate in aging cells due to their exposure to damaging stresses or expression of proteins with dominant conformations that tend towards aggregation. Such proteins can, however, be folded by ATP-dependent chaperones such as Hsp70 or small HSP such as Hsp27 that function independently of ATP and tend to form high-molecular-weight folding complexes. Each pathway plays a significant role in longevity through recognition and refolding of damaged proteins.

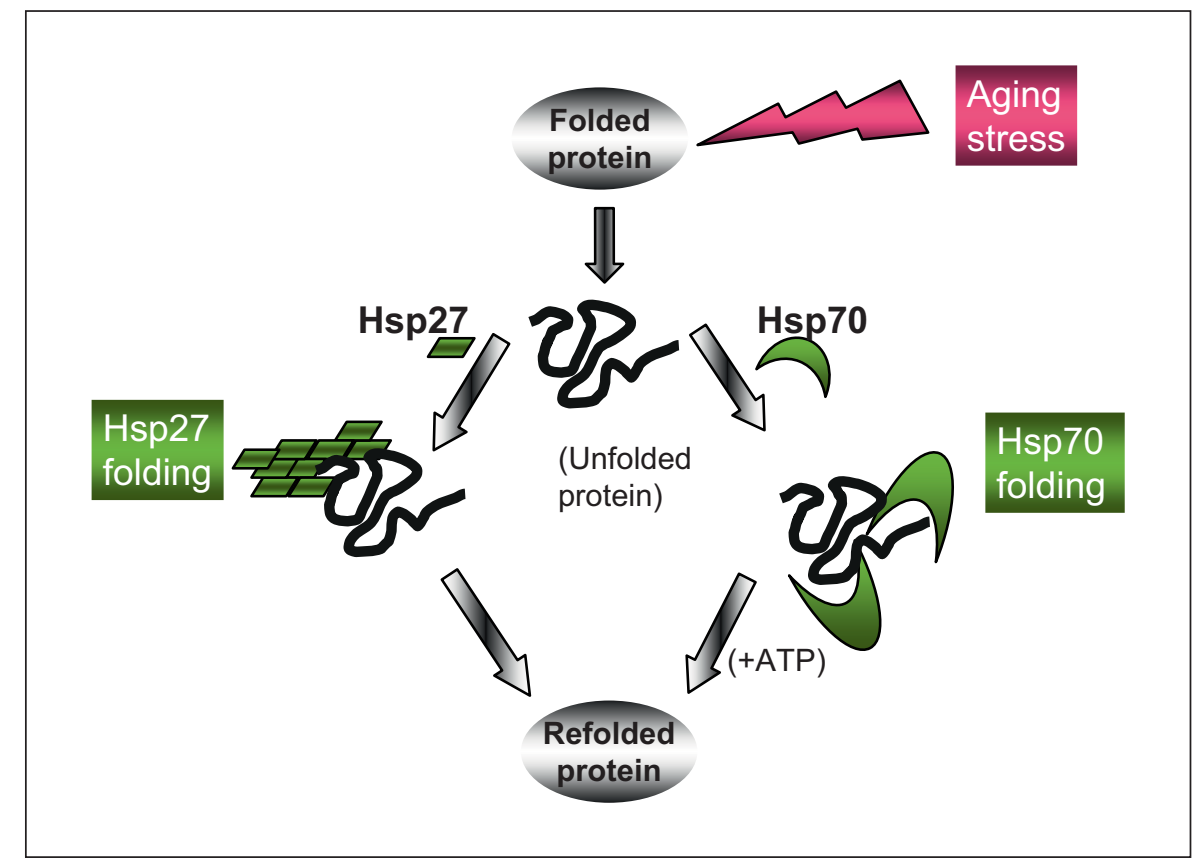

Molecular chaperones are essential in aging when damaged proteins or proteins undergoing aggregation due to dominant conformations that tend towards aggregation begin to accumulate. Such protein aggregation is deterred by HSPs [13]. Studies in Escherichia coli show that aggregation during heat shock involves a discrete group of unstable proteins whose aggregation is deterred by the E. coli HSP70 homolog DnaK [11]. Although such studies have not been carried out in mammalian cells, many key regulatory proteins such as kinases and transcription factors have unstable conformations that require constant chaperoning by Hsp90-containing complexes to retain activity [14]. This would strongly suggest that such essential proteins undergo selective loss when protein quality control mechanisms decline during aging.

\section{Molecular Chaperones and Proteases Combine to Mediate Protein Quality Control}

A second strategy for protein quality control involves degradation of damaged proteins by proteases. A compelling connection between the chaperone and protease pathways of quality control involves carboxyl terminus of HSP70-interacting protein (CHIP) which bridges the molecular chaperone and ubiquitin-proteasome protein degradation systems [15]. CHIP contains both a U box domain that permits it to conjugate ubiquitin to its sub- strates and a TPR domain for binding to Hsp70, Hsp90 and other TPR domain proteins. Significantly, CHIP plays a major role in longevity and CHIP inactivation in mice leads to a marked reduction in lifespan along with accelerated age-related pathophysiological phenotypes [16]. These include accelerated cellular senescence and increased incidence of oxidative stress [16]. The significance of CHIP in aging reflects the fact that the ubiquitin system participates in degradation of unfolded proteins by catalyzing the covalent tagging of proteins selected for degradation through the proteasome, a dedicated protein degradation machine. CHIP thus targets denatured proteins bound to Hsp70, Hsc70 or Hsp90 for degradation by catalyzing their ubiquitinylation.

Denatured proteins within aging cells may therefore suffer at least four distinct fates, based on the contrasting interactions between the damaged proteins, molecular chaperones and CHIP (fig. 2). Aggregates may continue to form progressively within aging cells as in pathway 1 , leading to formation of intracellular inclusions, impaired function, and death $[6,7]$. However, damaged proteins may enter pathway 2 after detection by denatured protein binding domains within Hsp70 (and Hsp90), be included within protein-chaperone complexes and be refolded (fig. 2, pathway 2). Such damaged proteins can also be processed by an alternative pathway (3) when the TPR acceptor site of HSP70 is bound to CHIP instead of HSP co-chaperones; in this case, the substrate protein is 
brought close to the U box domain of CHIP, becomes polyubiquitinylated and targeted to the proteasome for rapid degradation [10]. By competing with co-chaperones, such as Hop for the TPR acceptor sites on Hsp70 and Hsp90, CHIP inhibits the refolding pathway while other TPR domain proteins can in turn inhibit the CHIP pathway. There are also further levels of regulation of the pathways shown in figure 2 which are mediated through additional co-chaperones that bind to Hsp70 including Bag1-1, Bag-2 and HSPBP1 [16]. These proteins are candidates for mediating triage between the competing pathways of protein refolding and degradation and provide further layers of regulation that may become deregulated during aging.

\section{Chaperone-Mediated Autophagy}

Proteins can also be broken down during autophagy, a pathway of cell autodigestion that is observed in starvation. Two of its major variant forms, macroautophagy and chaperone-mediated autophagy (CMA), undergo age-dependent declines [17]. During CMA, Hsc70 recruits cytoplasmic proteins to the lysosome and mediates their entry into the lysosomal lumen permitting degradation [17] (fig. 2, pathway 4). CMA is stimulated by both starvation and oxidative stress and is responsible for degradation of the $30 \%$ of intracellular proteins that contain the KFERQ, CMA recognition motif [17]. CMA resembles CHIP-mediated proteasomal degradation in that damaged proteins form complexes containing Hsc70, Hsp40, Hop, Hsp90 and Bag-1 that presumably mediate CMA-substrate recognition and unfolding prior to lysosomal insertion [17]. Thereafter, the two processes diverge as substrates for CMA are delivered to lysosomal membrane receptor LAMP2A, are inserted into the lysosomal lumen and then are accepted in the lumen by a population of intralysosomal Hsc70 molecules. Both LAMP2A and Hsc70 levels are modulated during starvation and oxidative stress and levels of LAMP2A in particular undergo decline during senescence [17]. Decline in CMA is involved in Parkinson's disease and this may involve defective degradation of $\alpha$-synuclein [17].

\section{Aggresomes, Histone Deacetylases and Aging}

When the capacity of cells to refold or degrade misfolded proteins as in figure 2 becomes saturated, another pathway can be used to clear protein aggregates. In this case, microaggregates formed in the cytoplasm are actively transported along microtubules to the centrosomes and accumulate in structures known as aggresomes [18]. Such aggresomes can then be disposed of by macroautophagy pathways or during asymmetric cell division, in which a single daughter cell inherits the aggresomes and is presumably sacrificed for the continued health of the other daughter [18]. This pathway appears to be regulated in mammalian cells by histone deacetylase 6 (HDAC6), an enzyme that can bind both to polyubiquitinylated proteins and to microtubules [19]. HDAC6 binds to Hsp90 and can regulate its chaperone functions through histone deacetylation [20]. Interestingly, HDAC6 is also coupled to HSF1 metabolism and is required for HSF1 activation during inhibition of proteasome activity. HDAC6 binding to polyubiquitin appears to trigger Hsp90 dissociation from HSF1 and its activation. Thus HDAC6, in addition to its role in aggresome formation, participates in molecular chaperone synthesis [19]. A link between histone deacetylation, aggresomes and aging is indicated by findings in yeast which show that asymmetric cell division requires Sir2 and declines with age [1]. Both HDAC6 and the human Sir2 homolog Sirt1 are tubulin deacetylases, co-associate on microtubules and are involved in HSF1 activation [18, 21]. The histone deacetylase activities of HDAC6 and Sirt1 may thus couple protein quality control to the longevity pathways.

\section{Decline in the Heat Shock Response and Protein Quality Control with Aging}

Elevated HSF1 activity is tightly connected to longevity in flies and nematodes and HSF1 is involved in the transcriptional response to starvation (fig. 3) [22]. Inhibition of HSF1 expression or function decreases lifespan while extra copies of HSF1 expand the lifespan of C. elegans [22]. HSF1 is essential for starvation-induced longevity as well as in responses to heat shock and oxidative stress [23]. Starvation coordinately activates Sir2 and HSF1 activity in C. elegans, a finding which is concordant with recent studies showing that mammalian Sirt1 activates HSF1 [21]. It is not known, however, whether the second longevity pathway triggered by starvation, a decline in signaling through the insulin/IGF-1/mTOR pathway, contributes to HSF1 activation in mammals. Aging in mammalian organisms involves degeneration of HSP expression with time and the loss of resistance to cellular oxidants which is confluent with the studies in C. elegans and Drosophila [22]. Enfeeblement of the heat 
Fig. 2. Quality control, salvage and disposal pathways for damaged proteins. Proteins acquire multiple forms of damage over the lifetime of the organism leading to unfolding. Such alterations then trigger protein aggregation and formation of inclusion bodies (as in pathway 1). Denatured and aggregated proteins can, however, be recognized by HSPs that bind exposed hydrophobic domains in the misfolded proteins and lead to salvage and refolding of the denatured protein directly through pathway 2. However, unfolded proteins may persist in the cytoplasm and enter a third pathway (3) through binding of the Hsp70 to CHIP. Recruitment of CHIP leads to ubiquitinylation of the damaged protein and degradation by the proteasome. Pathways 2 and 3 thus compete for the denatured proteins. Another protein degradation pathway (4) is also mediated by molecular chaperones. A subset of damaged proteins is bound to Hsp70 family member Hsc70 and transported to the lysosome for degradation in CMA.

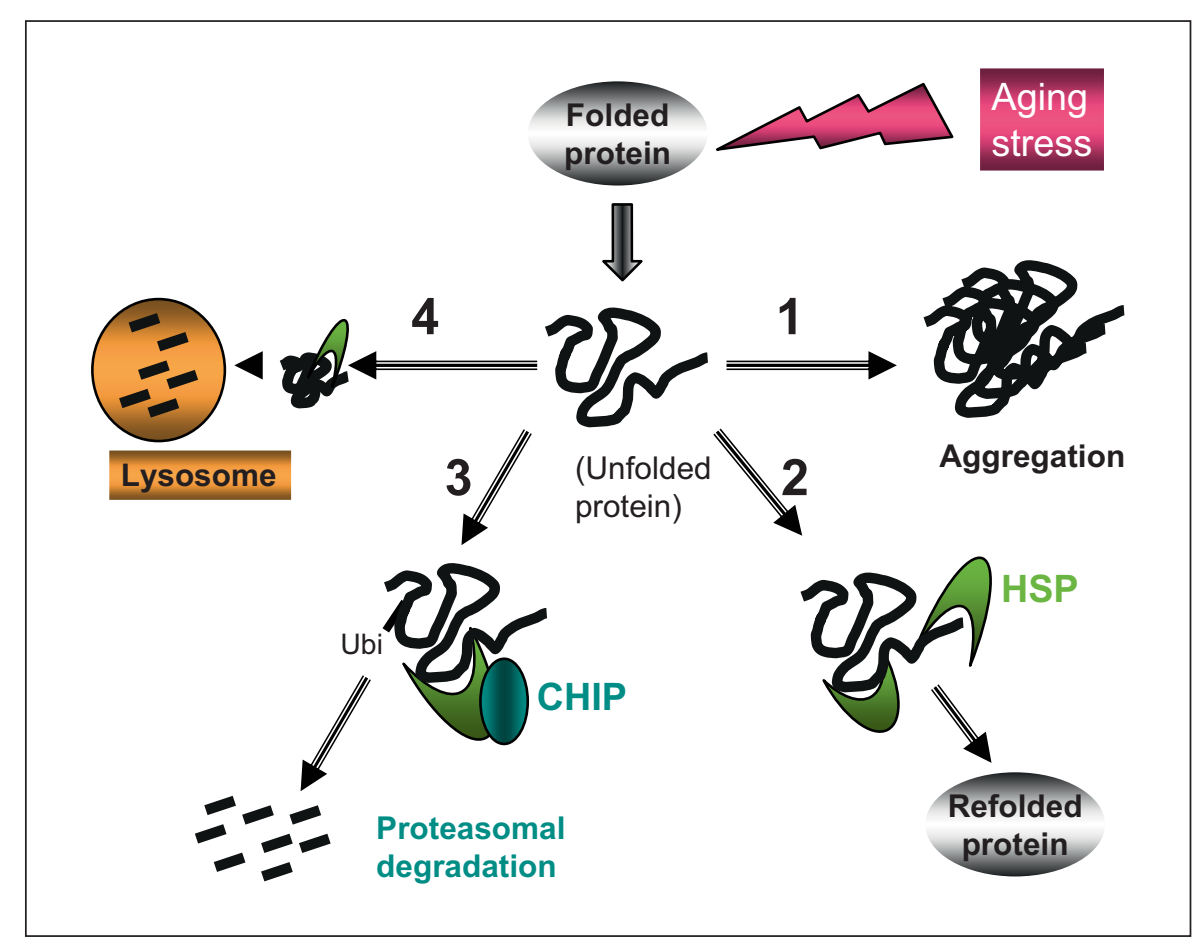

shock response during aging involves reduced transcriptional activation of small heat shock protein genes (sHSP: HSP16.1; HSP16.49; HSP12.6) in C. elegans [22]. Indeed HSP-mediated longevity involves protection of neuronal function and motor function and RNAi antagonism of HSF1 expression accelerates the onset of polyglutamine aggregation in C. elegans [22]. The sHSPs (as well as Hsp70) play analogous roles in regulating longevity in Drosophila [24]. Inactivation of the sHSP family HSP22 gene in Drosophila markedly decreases lifespan and a similar decrease in lifespan is seen if only motor neurons are targeted, strongly implicating a critical role for these tissues in the effects of HSPs on longevity in flies [25]. The longevity-friendly effects of sHSPs may partially reflect their capacity to oppose the toxicity associated with oxidative stress [26]. sHSPs play a similar role in humans and recent findings show protective effects for HSP27 in Huntington's disease [27]. Naturally-occurring polymorphisms in HSP22 and HSP27 are also observed in motor neuron neuropathy [28]. In addition, mutations in Hsp27 are seen in patients with Carcot-Marie-Tooth neuromuscular disease and distal hereditary motor neuropathy [29].

Members of the HSF family are found in all eukaryotes [30]. Mammalian cells contain at least 3 HSF family members, HSF1, HSF2 and HSF4 [30]. Neurons appear to be deficient in the heat shock response while retaining ability to express such HSF proteins [31]. Furthermore, HSF1 fails to be activated in motor neurons even when microinjected with plasmids encoding an HSF1 expression vector, suggesting a block to the HSF1 signal transduction pathways in these cells [32].

HSF1 is repressed under non-stress conditions by a complex containing Hsp90 and other proteins (fig. 4). In this inactive state, HSF1 is a monomer that lacks the ability to bind cis-acting heat shock elements (HSE) in the promoters of HSP genes (fig. 4) [30]. Protein stress results in conversion of HSF1 from inactive monomer to DNAbinding trimer and remodeling of the inhibitory molecular chaperone complex [30]. Activation of HSF1 by heat shock is a multi-step process, involving multiple inducible phosphorylation, dephosphorylation, acetylation and deacetylation steps, the sum of which results in the transcription of HSP genes (fig. 4). Extracellular signal input during heat shock involves tyrosine phosphorylation upstream of HSF1, involving the receptor tyrosine kinase HER2 and launching downstream signaling cascades through intracellular kinase Akt [33]. Akt regulates HSF1 at least in part through modulating its association with the phosphoserine binding scaffold protein 14-3-3 [33]. Nuclear exclusion and repression of HSF1 involves dual phosphorylation by protein kinases ERK1 and GSK3 
(the latter a downstream target of the HER2/Akt pathway) and subsequent recruitment of 14-3-3 [34]. Of emerging importance for HSF1 activation by longevity stimuli, HSF1 activity is stimulated by the HDAC Sirt1, directly coupling HSF1 to the conserved longevity pathways [21] (fig. 3). HSF1 is also regulated by proteasomal stress and decline in proteasome function or increases in proteasome substrates trigger the response through HDAC6 as discussed above [19, 20]. Although HDAC activity is involved in activation, the exact role of acetylation and deacetylation in HSF1 regulation is still not completely defined.

Another pathway for HSF1 activation has recently emerged involving a link to ribosomal function. Binding to the translation elongation factor eEF1A leads to HSF1 trimerization and is essential for activation [35]. HSF1 activation during heat shock occurs only when eEF1A is complexed to a non-coding RNA, heat shock RNA-1 (HSR1) [35]. eEF1A is an inhibitor of apoptotic death in muscle and neuronal tissues suggesting a link to aging [36]. Unraveling the relative roles of eEF1A in regulating mRNA translation or HSF1 activity may permit assessment of the potential role of this factor in HSF1-mediated aging.

Decreases in the rate of HSP transcription and HSF1 activity during aging occur in many tissues $[8,9]$. Failure of the heat shock response with time in neuronal tissues was first ascribed to decreased HSF1 expression and declining capacity of cells to form DNA binding HSF1 trimers [reviewed in 32]. Decreases in HSF1 levels during aging could thus place the response beneath the threshold essential for activation. Subsequent studies also indicated a further defect in HSF regulation in cultured motor neurons and Batalan et al. [32] showed that, while HSF1 is not activated in cultured neuronal cells even under conditions of HSF1 overexpression, a construct lacking the regulatory domain could be activated. This domain is a major protein interaction region, and double phosphorylation of this sequence by ERK1 and GSK3 leads to HSF1 repression through recruitment of 14-3-3 and nuclear exclusion [34, 37]. Nuclear exclusion results in loss of HSF1 from HSP gene promoters and repression of HSP transcription [34]. Abnormal increases in GSK3 levels occur in Alzheimer's disease, an effect (associated with tau aggregation) which may lead to HSF1 repression [38]. 14-3-3 levels also become elevated in areas of the brain subjected to prion diseases, and the appearance of 14-3-3 proteins in the cerebrospinal fluid is characteristic of some neurodegenerative diseases. Thus, age-related changes in GSK3 and 14-3-3 levels and activities could be

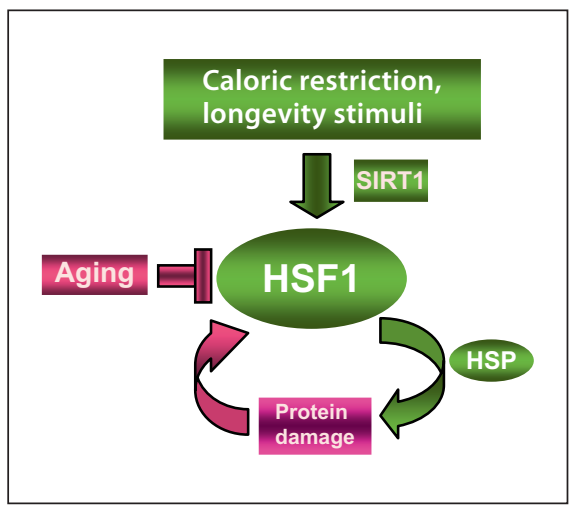

Fig. 3. HSF1 in longevity and aging. HSF1 is activated when damaged proteins derepress its activity, permit HSP expression and stimulate repair of protein damage. HSF1 can also be directly stimulated by longevity stimuli such as the histone deacetylase SIRT1 that directly activates HSF1 by deacetylation and fosters longevity. Aging, however, is associated with a gradual decline in potency of the heat shock response and this may prevent repair of protein damage, leading to degeneration and cell death.

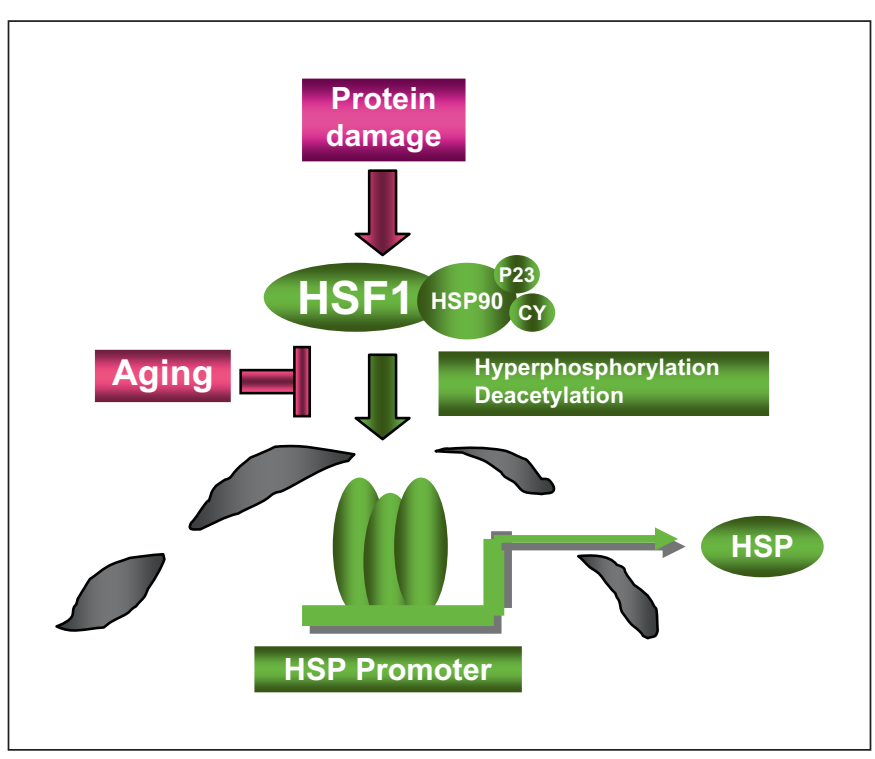

Fig. 4. HSF1 regulation by damaged proteins. Damaged proteins may accumulate in cells due to exposure to proteotoxins or decline in protein degradation pathways. Such damaged proteins cause the release of HSF1 from inert cytoplasmic complexes containing Hsp90 and its co-chaperones including p23 and cyclophilins (CY), trimerization, migration to the nucleus and binding to HSP gene promoters. Full HSF1 activation involves the triggering of a complex network of posttranslational modifications that lead to hyperphosphorylation and deacetylation of key residues. Loss of HSF1 inducibility in aging may involve alterations in HSF1-chaperone complexes or in signal transduction pathways upstream of enzymes that regulate hyperphosphorylation or deacetylation. 
involved in the progressive decline of the heat shock response. HSF1 is also regulated by CHIP [15]. It may be significant that CHIP and Hsc70 cooperate with another ubiquitin E3 ligase, Parkin, in the degradation of the receptor Pael-R and that defects in this system mediate changes involved in some cases of Parkinson's disease [39]. It would thus be instructive to examine age-related CHIP activity in relation to Parkin inactivation in aging cells [39]. In addition, when CHIP associates with Hsp70 and Hsp90 through its TPR domains it can lead to polyubiquitinylation of the molecular chaperones themselves in addition to their polypeptide cargoes [15]. Dysregulation of HSP-CHIP interactions and altered HSP degradation may thus also underlie some of the changes in HSP levels that accompany aging [16]. CHIP also associates with the expanded polyglutamine repeats that mediate aggregation during aging, and sequestration of CHIP by high concentrations of proteins bearing polyglutamine repeats may repress HSF1 during aging [40].

\section{The Prospect of HSP Inducers as Drugs to Ameliorate Aging}

From the preceding discussion, it is evident that a decline in molecular chaperone expression accompanies aging. Thus, chemical induction of HSP expression might be a promising approach for ameliorating the tissue degeneration accompanying aging and perhaps in increasing longevity. HSF1 activation and HSP expression occur in cells exposed to a wide range of agents that lead to accumulation of protein damage, such as heat shock, proteasome inhibitors, heavy metals, ethanol, arsenite and Hsp90 inhibitors $[2,9,19,20]$. While most of these treatments cause a wide range of toxicities to go along with HSP induction, making them unsuitable as selective agents, Hsp90 inhibitors are under investigation as potential drugs for reversing neurodegeneration [15]. Hsp90 inhibitors that are currently being developed as anticancer agents derepress HSF1 and lead to new HSP synthesis [14]. However, in addition to Hsp70 induction, current Hsp90 inhibitors cause degradation of a wide range of receptors, kinases and transcription factors and are likely to induce significant complications [14]. New formulations of such drugs will thus be required. The finding that HSF1 is regulated by SIRT1 suggests that compounds that activate this molecule such as resveratol or dihydrocoumarin include HSF1 activation as part of their pro-longevity modus [1, 21]. Future pursuit of these classes of compounds in the search for HSP-inducing drugs thus seems indicated. Drugs with more profound HSF1 specificity may await understanding of the regulation of transcription by HSF1 (fig. 3). Transcriptional regulation by HSF1 is still understood only in broad outline at the molecular level and more thorough characterization of its signaling and regulatory mechanisms may open the way for design of novel pharmaceuticals. Pharmaceutical targeting of the HSF1/HSP system is taking place at a rapid rate now that roles in cancer and aging have been identified, and prompt advances may be expected [14].

\section{Conclusions}

There is little doubt that HSF1 and the pathways of protein quality control are closely coupled to the mechanisms that ensure longevity in non-mammalian and mammalian species. There is also strong evidence indicating that HSPs protect a range of human cell types from protein damage and deter loss of function and cell death during aging by ensuring protein quality and inhibiting programmed cell death pathways. Increased levels of HSP are associated with longevity, decreased levels with degeneration and death. The role of the heat shock response in deterring the aging of individual tissues may vary in relation to the degree of accumulation of chaperone clients such as protein aggregates in the tissue and the relative potency of HSF1 regulation. The stage is thus set for a more thorough interrogation of the role of this system in aging and longevity in mammalian models and human patients.

\section{Acknowledgement}

This work was supported by NIH research grants R01CA047407 and R01CA094397.

\footnotetext{
References $>1$ Westphal CH, Dipp MA, Guarente L: A therapeutic role for sirtuins in diseases of aging? Trends Biochem Sci 2007;32:555-560.

$\checkmark 2$ Lindquist S, Craig EA: The heat shock proteins. Annu Rev Genet 1988;22:631-637.

-3 Garrido C, Brunet M, Didelot C, Zermati Y, Schmitt E, Kroemer G: Heat shock proteins 27 and 70: anti-apoptotic proteins with tumorigenic properties. Cell Cycle 2006;5: 2592-2601.

4 Ellis RJ: Protein misassembly: macromolecular crowding and molecular chaperones. Adv Exp Med Biol 2007;594:1-13.
} 
5 Sherman MY, Goldberg AL: Cellular defenses against unfolded proteins: a cell biologist thinks about neurodegenerative diseases. Neuron 2001;29:15-32.

6 Winklhofer KF, Tatzelt J, Haass C: The two faces of protein misfolding: gain- and lossof-function in neurodegenerative diseases. EMBO J 2008;27:336-349.

7 Hands S, Sinadinos C, Wyttenbach A: Polyglutamine gene function and dysfunction in the ageing brain. Biochim Biophys Acta 2008;1779:507-521.

$\checkmark 8$ Kayani AC, Morton JP, McArdle A: The exercise-induced stress response in skeletal muscle: failure during aging. Appl Physiol Nutr Metab 2008;33:1033-1041.

-9 Gagliano N, Grizzi F, Annoni G: Mechanisms of aging and liver functions. Dig Dis 2007;25:118-123.

10 Calderwood SK: Molecular Chaperones and the Ubiquitin Proteasome System in Aging. New York, Nova, 2007.

11 Mayer MP, Bukau B: Hsp70 chaperones: cellular functions and molecular mechanism. Cell Mol Life Sci 2005;62:670-684.

12 D'Andrea LD, Regan L: TPR proteins: the versatile helix. Trends Biochem Sci 2003;28: 655-662.

13 Hut HM, Kampinga HH, Sibon OC: Hsp70 protects mitotic cells against heat-induced centrosome damage and division abnormalities. Mol Biol Cell 2005;16:3776-3785.

-14 Gray PJ Jr, Prince T, Cheng J, Stevenson MA, Calderwood SK: Targeting the oncogene and kinome chaperone CDC37. Nat Rev Cancer 2008;8:491-495.

15 Marques C, Guo W, Pereira P, Taylor A, Patterson C, Evans PC, Shang F: The triage of damaged proteins: degradation by the ubiquitin-proteasome pathway or repair by molecular chaperones. FASEB J 2006;20:741743.

16 Min JN, Whaley RA, Sharpless NE, Lockyer P, Portbury AL, Patterson C: CHIP deficiency decreases longevity, with accelerated aging phenotypes accompanied by altered protein quality control. Mol Cell Biol 2008;28: 4018-4025.

17 Dice JF: Chaperone-mediated autophagy. Autophagy 2007;3:295-299.

18 Rujano MA, Bosveld F, Salomons FA, Dijk F, van Waarde MA, van der Want JJ, de Vos RA, Brunt ER, Sibon OC, Kampinga HH: Polarised asymmetric inheritance of accumulated protein damage in higher eukaryotes. PLoS Biol 2006;4:e417.
19 Boyault C, Zhang Y, Fritah S, Caron C, Gilquin B, Kwon SH, Garrido C, Yao TP, Vourc'h C, Matthias P, Khochbin S: HDAC6 controls major cell response pathways to cytotoxic accumulation of protein aggregates. Genes Dev 2007;21:2172-2181.

20 Kovacs JJ, Murphy PJ, Gaillard S, Zhao X, Wu JT, Nicchitta CV, Yoshida M, Toft DO, Pratt WB, Yao TP: HDAC6 regulates Hsp90 acetylation and chaperone-dependent activation of glucocorticoid receptor. Mol Cell 2005; 18:601-607.

21 Westerheide SD, Anckar J, Stevens SM Jr, Sistonen L, Morimoto RI: Stress-inducible regulation of heat shock factor 1 by the deacetylase SIRT1. Science 2009;323:1063-1066.

22 Hsu AL, Murphy CT, Kenyon C: Regulation of aging and age-related disease by DAF-16 and heat-shock factor. Science 2003;300: 1142-1145.

23 Steinkraus KA, Smith ED, Davis C, Carr D, Pendergrass WR, Sutphin GL, Kennedy BK, Kaeberlein M: Dietary restriction suppresses proteotoxicity and enhances longevity by an hsf-1-dependent mechanism in Caenorhabditis elegans. Aging Cell 2008;7:394-404.

-24 Kurapati R, Passananti HB, Rose MR, Tower J: Increased hsp22 RNA levels in Drosophila lines genetically selected for increased longevity. J Gerontol A Biol Sci Med Sci 2000;55: B552-559.

25 Morrow G, Samson M, Michaud S, Tanguay RM: Overexpression of the small mitochondrial Hsp22 extends Drosophila life span and increases resistance to oxidative stress. FASEB J 2004; 18:598-599.

26 Arrigo AP: The cellular 'networking' of mammalian Hsp27 and its functions in the control of protein folding, redox state and apoptosis. Adv Exp Med Biol 2007;594:1426.

27 Wyttenbach A, Sauvageot O, Carmichael J, Diaz-Latoud C, Arrigo AP, Rubinsztein DC: Heat shock protein 27 prevents cellular polyglutamine toxicity and suppresses the increase of reactive oxygen species caused by huntingtin. Hum Mol Genet 2002;11:11371151.

28 Benndorf R, Welsh MJ: Shocking degeneration. Nat Genet 2004;36:547-548.

29 Evgrafov OV, Mersiyanova I, Irobi J, Van Den Bosch L, Dierick I, Leung CL, Schagina O, Verpoorten N, Van Impe K, Fedotov V, Dadali E, Auer-Grumbach M, Windpassinger C, Wagner K, Mitrovic Z, Hilton-Jones D, Talbot K, Martin JJ, Vasserman N, Tverskaya S, Polyakov A, Liem RK, Gettemans J, Robberecht W, De Jonghe P, Timmerman V: Mutant small heat-shock protein 27 causes axonal Charcot-Marie-Tooth disease and distal hereditary motor neuropathy. Nat Genet 2004;36:602-606.
$30 \mathrm{Wu}$ C: Heat shock transcription factors: structure and regulation. Annu Rev Cell Dev Biol 1995;11:441-469.

-31 Kaarniranta K, Oksala N, Karjalainen HM, Suuronen T, Sistonen L, Helminen HJ, Salminen A, Lammi MJ: Neuronal cells show regulatory differences in the hsp70 gene response. Brain Res Mol Brain Res 2002;101: 136-140.

- 32 Batulan Z, Shinder GA, Minotti S, He BP, Doroudchi MM, Nalbantoglu J, Strong MJ, Durham HD: High threshold for induction of the stress response in motor neurons is associated with failure to activate HSF1. J Neurosci 2003;23:5789-5798.

-33 Khaleque MA, Bharti A, Sawyer D, Gong J, Benjamin IJ, Stevenson MA, Calderwood SK: Induction of heat shock proteins by heregulin $\beta_{1}$ leads to protection from apoptosis and anchorage-independent growth. Oncogene 2005;24:6564-6573.

34 Wang X, Grammatikakis N, Siganou A, Calderwood SK: Regulation of molecular chaperone gene transcription involves the serine phosphorylation, 14-3-3 epsilon binding, and cytoplasmic sequestration of heat shock factor 1. Mol Cell Biol 2003;23:60136026.

35 Shamovsky I, Ivannikov M, Kandel ES, Gershon D, Nudler E: RNA-mediated response to heat shock in mammalian cells. Nature 2006;440:556-560.

-36 Chang R, Wang E: Mouse translation elongation factor eEF1A-2 interacts with Prdx-I to protect cells against apoptotic death induced by oxidative stress. J Cell Biochem 2007;100: 267-278.

37 Chu B, Soncin F, Price BD, Stevenson MA, Calderwood SK: Sequential phosphorylation by mitogen-activated protein kinase and glycogen synthase kinase 3 represses transcriptional activation by heat shock factor-1. J Biol Chem 1996;271:30847-30857.

38 Bhat RV, Budd Haeberlein SL, Avila J: Glycogen synthase kinase 3: a drug target for CNS therapies. J Neurochem 2004;89:1313-1317.

39 Takahashi R, Imai Y: Pael receptor, endoplasmic reticulum stress, and Parkinson's disease. J Neurol 2003;250(suppl 3):III25III29.

40 Jana NR, Dikshit P, Goswami A, Kotliarova S, Murata S, Tanaka K, Nukina N: Co-chaperone CHIP associates with expanded polyglutamine protein and promotes their degradation by proteasomes. J Biol Chem 2005; 280:11635-11640. 